\title{
Approximate Periodic Solution for the Nonlinear Helmholtz-Duffing Oscillator via Analytical Approaches
}

\author{
A. Mirzabeigy, ${ }^{1,2}$ M. K. Yazdi, ${ }^{1}$ and M. H. Nasehi ${ }^{2}$ \\ ${ }^{1}$ School of Mechanical Engineering, Iran University of Science and Technology, Narmak, Tehran 16846, Iran \\ ${ }^{2}$ Department of Mechanical Engineering, Amirkabir University of Technology, Hafez Avenue, Tehran 15914, Iran
}

Correspondence should be addressed to A. Mirzabeigy; alborz.mi1987@yahoo.com

Received 1 June 2014; Accepted 17 September 2014; Published 29 September 2014

Academic Editor: Anh-Huy Phan

Copyright (c) 2014 A. Mirzabeigy et al. This is an open access article distributed under the Creative Commons Attribution License, which permits unrestricted use, distribution, and reproduction in any medium, provided the original work is properly cited.

\begin{abstract}
The conservative Helmholtz-Duffing oscillator is analyzed by means of three analytical techniques. The max-min, second-order of the Hamiltonian, and the global error minimization approaches are applied to achieve natural frequencies. The obtained results are compared with the homotopy perturbation method and numerical solutions. The results show that second-order of the global error minimization method is very accurate, so it can be widely applicable in engineering problems.
\end{abstract}

\section{Introduction}

Mathematical modeling and frequency analysis of the nonlinear vibrational systems are an important and interesting field of mechanics. A lot of researchers have worked in this field and have proposed a lot of methods for demonstrating the dynamics responses of these systems [1-4]. They have developed this field of science and have analyzed the responses of the nonlinear vibration problems such as Duffing oscillators [5-10], nonlinear dynamics of a particle on a rotating parabola [11], nonlinear oscillators with discontinuity [12], oscillators with noninteger order nonlinear connection [13], the plasma physics equation [14], and van der Pol oscillator $[15,16]$. The Helmholtz-Duffing equation is a nonlinear problem with the quadratic and cubic nonlinear terms. Surveying the literature shows that this equation has wide applications in the engineering problems. For example, due to different vibration behavior of functionally graded materials (FGMs) at positive and negative amplitudes, the governing equations of FGM beams, plates, and shells are conduced to a second-order nonlinear ordinary equation with quadratic and cubic nonlinear terms [17-20]. Moreover, Sharabiani and Yazdi [21] obtained a Helmholtz-Duffing type equation within studying of nonlinear free vibrations of functionally graded nanobeams with surface effects. On the other hand, they revealed application of this equation in FG nanostructures.

In this paper, the frequency-amplitude relationship of the conservative Helmholtz-Duffing oscillator is obtained by means of the max-min [22-26], Hamiltonian [27-31], and global error minimization methods [32-35]. The Hamiltonian approach is a kind of energy method and is proposed by $\mathrm{He}$ [27]. It is a simple method and can be used for the conservative nonlinear equations. Recently, it is applied for dynamic analysis of an electromechanical resonator [36]. Moreover, Akbarzade and Khan [37] employed the secondorder Hamiltonian approach for nonlinear dynamic analysis of conservative coupled systems of mass-spring. The maxmin approach is made on the base of Chengtian's inequality [38]. It is a valuable method for obtaining the frequency responses of the nonlinear problems, and many researchers are attracted to use this method for studying the nonlinear systems. The global error minimization method is a modified type of variational approach and converts the nonlinear equation to an equivalent minimization problem.

The Helmholtz-Duffing equation is analyzed by many researchers. For instance, Leung and Guo [39] have applied the homotopy perturbation method (HPM) to this equation 
and have obtained accurate responses. Askari et al. [40] studied approximate periodic solutions of this equation using He's energy balance method (HEBM) and He's frequencyamplitude formulation (HFAF). Akbarzade et al. [41] used the first-order of the Hamiltonian approach and coupled homotopy-variational formulation to study the periodic solutions of the Helmholtz-Duffing oscillator; they also discussed the stability of the system for selected constant parameters. Recently, Li et al. [42] determined limit cycles and homoclinic orbits of this oscillator via a generalized harmonic function perturbation method. In the next sections, the max-min, the Hamiltonian, and the global error minimization methods are applied for evaluating the dynamics responses of the Helmholtz-Duffing equation. The results of these methods are compared with the exact ones and HPM solutions.

\section{Solution Procedure}

Let us consider the Helmholtz-Duffing equation

$$
\begin{gathered}
\ddot{u}+u+(1-\sigma) u^{2}+\sigma u^{3}=0, \quad u(0)=A, \\
\dot{u}(0)=0 .
\end{gathered}
$$

The response oscillates between an asymmetric limit zone $[-b, A]$, where both $A$ and $b$ are positive. One can determine $b$ in the form of [39]

$$
\begin{aligned}
b= & \frac{1}{9 A}(3 A \sigma+4-4 A)+\frac{1}{9 A} \Delta^{1 / 3} \\
& -\frac{2}{9 A}\left(9 A^{2} \sigma^{2}+6 A \sigma-6 A \sigma^{2}+43 \sigma-8-8 \sigma^{2}\right) \Delta^{-1 / 3},
\end{aligned}
$$

where

$$
\begin{aligned}
& \Delta=270 A^{2} \sigma^{2}(1+A \sigma-\sigma)-72 A \sigma\left(1+\sigma^{2}\right) \\
&-516 \sigma(1-\sigma)+64\left(1-\sigma^{3}\right)+630 A \sigma^{2} \\
&+54 \sigma[-12\left(1+\sigma^{2}\right)+16 A\left(a+1+A \sigma^{4}-\sigma^{3}\right) \\
&+ 78 A\left(1+A^{3} \sigma-A^{3} \sigma^{2}\right)-8 A^{3} \sigma\left(1-\sigma^{3}\right) \\
&-172 A^{2} \sigma\left(1+\sigma^{2}\right)-120 A \sigma(1-\sigma)+9 A^{4} \sigma^{2} \\
& \times\left(1+5 A \sigma+10 \sigma+3 A^{2} \sigma^{2}-5 A \sigma^{2}+\sigma^{2}\right) \\
&+\left.447 A^{2} \sigma^{2}\right]^{1 / 2} .
\end{aligned}
$$

2.1. Max-Min Approach (MMA). We can rewrite (1) in the following form:

$$
\ddot{u}+\left(1+(1-\sigma) u+\sigma u^{2}\right) u=0 .
$$

By choosing $u(t)=A \cos (\omega t)$ as a trial function that satisfied the initial conditions, the maximum and minimum values of $1+(1-\sigma) u+\sigma u^{2}$ can be calculated with Maple software as $1+A-\sigma A+\sigma A^{2}$ and $-\left(\sigma^{2}-6 \sigma+1\right) / 4 \sigma$, respectively, so we can write

$$
-\frac{\sigma^{2}-6 \sigma+1}{4 \sigma}<\omega^{2}<1+A-\sigma A+\sigma A^{2} .
$$

By using Chengtian interpolation [38], we have

$$
\begin{aligned}
\omega^{2} & =\frac{-m\left(\left(\sigma^{2}-6 \sigma+1\right) / 4 \sigma\right)+n\left(1+A-\sigma A+\sigma A^{2}\right)}{m+n} \\
& =1-(1-k)\left(\frac{\sigma^{2}-2 \sigma+1}{4 \sigma}\right)+k\left(A-\sigma A+\sigma A^{2}\right),
\end{aligned}
$$

where $m, n$ are weighting factor, $k=n /(m+n)$. Therefore the frequency can be approximated as

$$
\omega=\sqrt{1+(1-k)\left(-\frac{\sigma^{2}-2 \sigma+1}{4 \sigma}\right)+k\left(A-\sigma A+\sigma A^{2}\right)} .
$$

Then the solution of (1) can be written:

$$
\begin{array}{r}
u(t)=A \cos \left(\left(1-(1-k)\left(\frac{\sigma^{2}-2 \sigma+1}{4 \sigma}\right)\right.\right. \\
\left.\left.+k\left(A-\sigma A+\sigma A^{2}\right) t\right)^{1 / 2}\right) .
\end{array}
$$

By using the approximate solution, (4) can be written in the following form:

$$
\begin{aligned}
\ddot{u}+ & \left(1-(1-k)\left(\frac{\sigma^{2}-2 \sigma+1}{4 \sigma}\right)+k\left(A-\sigma A+\sigma A^{2}\right)\right) u \\
= & -(1-k)\left(\frac{\sigma^{2}-2 \sigma+1}{4 \sigma}\right) u+k\left(A-\sigma A+\sigma A^{2}\right) u \\
& -(1-\sigma) u^{2}-\sigma u^{3} .
\end{aligned}
$$

If by chance (8) is the exact solution of (1), then the righthand side of (9) vanishes. According to [22], we set

$$
\begin{gathered}
\int_{0}^{T / 4}\left((k-1)\left(\frac{\sigma^{2}-2 \sigma+1}{4 \sigma}\right) u+k\left(A-\sigma A+\sigma A^{2}\right) u\right. \\
\left.-(1-\sigma) u^{2}-\sigma u^{3}\right) \cos (\omega t) d t=0,
\end{gathered}
$$

where $T=2 \pi / \omega$. By substituting (8) into (10), we obtain the following expression for $k$ :

$$
k=\frac{1}{3} \frac{3 \pi-6 \pi \sigma+3 \pi \sigma^{2}+32 A \sigma-32 A \sigma^{2}+9 \pi A^{2} \sigma^{2}}{\pi\left(1-2 \sigma+\sigma^{2}+4 A \sigma-4 A \sigma^{2}+4 A^{2} \sigma^{2}\right)} .
$$

Substituting (11) into (7) and after some simplification, we have

$$
\omega=\sqrt{1+\frac{8}{3 \pi} A-\frac{8}{3 \pi} A \sigma+\frac{3}{4} A^{2} \sigma} .
$$




\subsection{Hamiltonian Approach}

2.2.1. The First-Order Hamiltonian Approach (FHA). The Hamiltonian of (1) is constructed as

$$
H=\frac{1}{2} \dot{u}^{2}+\frac{1}{2} u^{2}+\frac{1}{3}(1-\sigma) u^{3}+\frac{1}{4} \sigma u^{4} .
$$

Assume the first approximate solution of (1) as

$$
u(t)=A \cos (\omega t) .
$$

Then integrating (13) with respect to time from 0 to $T / 4$, we have

$$
\widetilde{H}(u)=\int_{0}^{T / 4}\left(\frac{1}{2} \dot{u}^{2}+\frac{1}{2} u^{2}+\frac{1}{3}(1-\sigma) u^{3}+\frac{1}{4} \sigma u^{4}\right) d t .
$$

Substituting (14) in (15) yields

$$
\begin{aligned}
\widetilde{H}(u)=\int_{0}^{T / 4}\left(\frac{1}{2} A^{2} \omega^{2} \sin ^{2}(\omega t)+\frac{1}{2} A^{2} \cos ^{2}(\omega t)\right. \\
\left.\quad+\frac{1}{3}(1-\sigma) A^{3} \cos ^{3}(\omega t)+\frac{1}{4} \sigma A^{4} \cos ^{4}(\omega t)\right) d t \\
=\int_{0}^{\pi / 2}\left(\frac{1}{2} A^{2} \omega \sin ^{2}(t)+\frac{1}{2 \omega} A^{2} \cos ^{2}(t)\right. \\
\left.\quad+\frac{1}{3 \omega}(1-\sigma) A^{3} \cos ^{3}(t)+\frac{1}{4 \omega} \sigma A^{4} \cos ^{4}(t)\right) d t \\
=\frac{1}{576} \frac{A^{2}\left(72 \pi+128 A-128 A \sigma+27 \pi A^{2} \sigma+72 \pi \omega^{2}\right)}{\omega} .
\end{aligned}
$$

Set

$$
\begin{aligned}
& \frac{\partial}{\partial A}\left(\frac{\partial \widetilde{H}}{\partial(1 / \omega)}\right) \\
& \quad=\frac{1}{48} A\left(-12 \pi \omega^{2}+12 \pi+32 A-32 A \sigma+9 \pi A^{2} \sigma\right)=0 .
\end{aligned}
$$

Finally,

$$
\omega_{\mathrm{FHA}}=\sqrt{1+\frac{8}{3 \pi} A-\frac{8}{3 \pi} A \sigma+\frac{3}{4} A^{2} \sigma} .
$$

The result of first-order Hamiltonian and max-min approaches is alike.

2.2.2. The Second-Order Hamiltonian Approach (SHA). For the second-order Hamiltonian approach, we consider the following equation as the response of the system:

$$
u=a \cos (\omega t)+b \cos (3 \omega t) \text {. }
$$

Whereas (19) must satisfy the initial condition we have

$$
A=a+b .
$$

Substituting (19) into (15) yields

$$
\begin{aligned}
& \widetilde{H}(u)=\int_{0}^{T / 4}\left[\frac{1}{2} \omega^{2}(a \sin (\omega t)+3 b \sin (3 \omega t))^{2}\right. \\
& +\frac{1}{2}(a \cos (\omega t)+b \cos (3 \omega t))^{2} \\
& +\frac{1}{3}(1-\sigma)(a \cos (\omega t)+b \cos (3 \omega t))^{3} \\
& \left.+\frac{1}{4} \sigma(a \cos (\omega t)+b \cos (3 \omega t))^{4}\right] d t \\
& \widetilde{H}(u)=\int_{0}^{\pi / 2}\left[\frac{1}{2} \omega(a \sin (t)+3 b \sin (3 t))^{2}\right. \\
& +\frac{1}{2 \omega}(a \cos (t)+b \cos (3 t))^{2} \\
& +\frac{1}{3 \omega}(1-\sigma)(a \cos (t)+b \cos (3 t))^{3} \\
& \left.+\frac{1}{4 \omega} \sigma(a \cos (t)+b \cos (3 t))^{4}\right] d t \\
& \widetilde{H}(u)=\frac{1}{60480 \omega} \\
& \times\left(13440 a^{3}-4480 b^{3}-13440 a^{3} \sigma\right. \\
& -8046 a^{2} b \sigma+2835 \pi a^{4} \sigma-31104 a b^{2} \sigma \\
& +8064 a^{2} b+2835 \pi b^{4} \sigma+4480 b^{3} \sigma \\
& +31104 a b^{2}+3780 \pi a^{3} b \sigma+11340 \pi a^{2} b^{2} \sigma \\
& \left.+7560 \pi a^{2}+7560 \pi b^{2}\right)+\frac{\pi \omega}{8}\left(a^{2}+9 b^{2}\right) .
\end{aligned}
$$

Set

$$
\begin{aligned}
\frac{\partial}{\partial a}\left(\frac{\partial \widetilde{H}}{\partial(1 / \omega)}\right)= & -\frac{1}{4} \pi a \omega^{2}+\frac{2}{3} a^{2}-\frac{2}{3} a^{2} \sigma \\
& -\frac{4}{15} a b \sigma+\frac{3}{16} \pi a^{3} \sigma-\frac{18}{35} b^{2} \sigma+\frac{4}{15} a b \\
& +\frac{18}{35} b^{2}+\frac{3}{16} \pi a^{2} b \sigma+\frac{3}{8} \pi a b^{2} \sigma+\frac{1}{4} \pi a=0
\end{aligned}
$$

$$
\begin{aligned}
\frac{\partial}{\partial b}\left(\frac{\partial \widetilde{H}}{\partial(1 / \omega)}\right)= & -\frac{9}{4} \pi b^{2} \omega^{2}-\frac{2}{9} b^{2}-\frac{2}{15} a^{2} \sigma \\
& -\frac{36}{35} a b \sigma+\frac{2}{15} a^{2}+\frac{3}{16} \pi b^{3} \sigma+\frac{2}{9} b^{2} \sigma \\
& +\frac{36}{35} a b+\frac{1}{16} \pi a^{3} \sigma+\frac{3}{8} \pi a^{2} b \sigma+\frac{1}{4} \pi b=0 .
\end{aligned}
$$

Since finding a relation between different parameters from (22) is not easy, the value of other parameters is determined in Table 1 for several values of $\sigma$. 
TABLE 1: Frequency-amplitude relationships for several values of $\sigma$.

\begin{tabular}{lccc}
\hline$\sigma$ & $a$ & $b$ & $\omega_{\text {SHA }}$ \\
\hline 0.1 & $0.987791887 A$ & $0.012208112 A$ & $\sqrt{1+0.758436858 A+0.074106747 A^{2}}$ \\
0.3 & $0.986559761 A$ & $0.013440239 A$ & $\sqrt{1+0.589470841 A+0.222057234 A^{2}}$ \\
0.5 & $0.985302609 A$ & $0.014697391 A$ & $\sqrt{1+0.420742301 A+0.369650489 A^{2}}$ \\
0.7 & $0.984019498 A$ & $0.015980502 A$ & $\sqrt{1+0.252257249 A+0.516878382 A^{2}}$ \\
0.9 & $0.982709435 A$ & $0.017290564 A$ & $\sqrt{1+0.084021956 A+0.663732471 A^{2}}$ \\
1 & $0.982043978 A$ & $0.017956021 A$ & $\sqrt{1+0.737016661 A^{2}}$ \\
\hline
\end{tabular}

\subsection{Global Error Minimization Method}

2.3.1. The First-Order Global Error Minimization Method (FGEM). Based on standard procedure of modified variational approach, the minimization problem is

Minimize $E(\dot{u}, u, t)$

$$
=\int_{0}^{T}\left(\ddot{u}+u+(1-\sigma) u^{2}+\sigma u^{3}\right)^{2} d t, \quad T=\frac{2 \pi}{\omega} .
$$

For first-order approximation, consider a trial function as follows:

$$
u(t)=A \cos (\omega t) .
$$

Substituting (24) into (23) yields

Minimize $E(\dot{u}, u, t)$

$$
\begin{gathered}
=\frac{\pi A^{2}}{8 \omega}\left(5 A^{4} \sigma^{2}-12 A^{2} \sigma \omega^{2}+6 A^{2} \sigma^{2}+8 \omega^{4}\right. \\
\left.-16 \omega^{2}+6 A^{2}+8\right) .
\end{gathered}
$$

The solution of (25) could be found through

$$
\frac{\partial E(\dot{u}, u, t)}{\partial \omega}=0
$$

By some simplifications, the following equation is obtained:

$$
\begin{gathered}
-24 \omega^{4}+12 A^{2} \omega^{2} \sigma+16 \omega^{2}+5 A^{4} \sigma^{2} \\
+6 A^{2} \sigma^{2}+6 A^{2}+8=0 .
\end{gathered}
$$

One may find the first-order approximation by solving (27) as

$$
\begin{aligned}
& \omega_{\mathrm{FGEM}} \\
& \qquad \begin{array}{c}
=\frac{1}{6}\left(9 A^{2} \sigma\right. \\
+3\left(39 A^{4} \sigma^{2}+36 A^{2} \sigma^{2}+24 A^{2} \sigma\right. \\
\left.\left.+36 A^{2}+64\right)^{1 / 2}\right)^{1 / 2} .
\end{array}
\end{aligned}
$$

2.3.2. The Second-Order Global Error Minimization Method (SGEM). For the second-order approximation, consider a trial function as

$$
u(t)=a \cos (\omega t)+b \cos (3 \omega t) .
$$

Whereas (29) must satisfy the initial condition, we have

$$
A=a+b .
$$

Substituting (29) into (23) yields

$$
\text { Minimize } E(\dot{u}, u, t)
$$

$$
\begin{aligned}
=\frac{\pi}{8 \omega}( & 5 \sigma^{2} a^{6}-144 b^{2} \omega^{2} \\
& +45 \sigma^{2} a^{2} b^{4}+45 \sigma^{2} a^{4} b^{2}+24 \sigma^{2} a^{2} b^{2} \\
& +8 \sigma^{2} a^{3} b+6 \sigma^{2} b^{4}+5 \sigma^{2} b^{6}+8 a^{2} \omega^{4} \\
& +30 \sigma^{2} a^{3} b^{3}+24 a^{2} b^{2}-16 a^{2} \omega^{2} \\
& +8 a^{2}+8 b^{2}+6 a^{4}-240 a^{2} \omega^{2} \sigma b^{2} \\
& +15 \sigma^{2} a^{5} b-12 a^{4} \omega^{2} \sigma+6 \sigma^{2} a^{4} \\
& +648 b^{2} \omega^{4}+6 b^{4}-108 b^{4} \omega^{2} \sigma \\
& \left.+8 a^{3} b-48 a^{3} \omega^{2} \sigma b\right) .
\end{aligned}
$$

The solution of (31) could be found through

$$
\begin{aligned}
& \frac{\partial E(\dot{u}, u, t)}{\partial \omega}=0, \quad \frac{\partial E(\dot{u}, u, t)}{\partial a}=0, \\
& \frac{\partial E(\dot{u}, u, t)}{\partial b}=0,
\end{aligned}
$$

which yield the following:

$$
\begin{aligned}
\frac{\partial E(\dot{u}, u, t)}{\partial \omega} & \\
=-\frac{\pi}{8 \omega^{2}}( & 5 \sigma^{2} a^{6}+8 a^{3} b+45 \sigma^{2} a^{2} b^{4} \\
& +8 a^{2}+8 b^{2}+5 \sigma^{2} b^{6}+45 \sigma^{2} a^{4} b^{2} \\
& -24 a^{2} \omega^{4}+16 a^{2} \omega^{2}+6 b^{4} \\
& +6 a^{4}+24 \sigma^{2} a^{2} b^{2}+15 \sigma^{2} a^{5} b \\
& +30 \sigma^{2} a^{3} b^{3}-1944 b^{2} \omega^{4} \\
& +144 b^{2} \omega^{3}+6 \sigma^{2} a^{4}+6 \sigma^{2} b^{4} \\
& +48 a^{3} \omega^{2} \sigma b+240 a^{2} \omega^{2} \sigma b^{2} \\
+ & 8 \sigma^{2} a^{3} b+12 a^{4} \omega^{2} \sigma \\
& \left.+108 b^{4} \omega^{2} \sigma+24 a^{2} b^{2}\right)=0
\end{aligned}
$$


TABLE 2: Comparison between MMA, FHA, SHA, FGEM, and SGEM results with exact ones and HPM responses $(\sigma=0.5)$.

\begin{tabular}{|c|c|c|c|c|c|c|}
\hline$A$ & $T_{\text {exact }}$ & $\begin{array}{c}T_{\mathrm{MMA}}=T_{\mathrm{FHA}} \\
\text { (relative error \%) }\end{array}$ & $\begin{array}{c}T_{\mathrm{FGEM}} \\
\text { (relative error \%) }\end{array}$ & $\begin{array}{c}T_{\mathrm{SHA}} \\
\text { (relative error \%) }\end{array}$ & $\begin{array}{c}T_{\text {SGEM }} \\
\text { (relative error \%) }\end{array}$ & $\begin{array}{c}T_{\mathrm{HPM}}[39] \\
\text { (relative error \%) } \\
\end{array}$ \\
\hline 0.01 & 6.283133 & $\begin{array}{l}6.26977721 \\
(0.2125657)\end{array}$ & $\begin{array}{l}6.28305278 \\
(0.0012767)\end{array}$ & $\begin{array}{c}6.2698934 \\
(0.2107154)\end{array}$ & $\begin{array}{l}6.28305278 \\
(0.0012767)\end{array}$ & $\begin{array}{c}6.283132 \\
(0.0000159)\end{array}$ \\
\hline 0.05 & 6.281851 & $\begin{array}{l}6.21470929 \\
(1.0688203) \\
\end{array}$ & $\begin{array}{l}6.27987482 \\
(0.0314585) \\
\end{array}$ & $\begin{array}{l}6.21530801 \\
(1.0592895) \\
\end{array}$ & $\begin{array}{l}6.27987515 \\
(0.0314630) \\
\end{array}$ & $\begin{array}{c}6.281831 \\
(0.0003184) \\
\end{array}$ \\
\hline 0.1 & 6.277721 & $\begin{array}{l}6.14291226 \\
(2.1474152)\end{array}$ & $\begin{array}{l}6.26997826 \\
(0.1233367)\end{array}$ & $\begin{array}{l}6.14414741 \\
(2.1277402)\end{array}$ & $\begin{array}{l}6.26998356 \\
(0.1234043)\end{array}$ & $\begin{array}{c}6.277642 \\
(0.0012584)\end{array}$ \\
\hline 10 & 1.004172 & $\begin{array}{c}0.9610403 \\
(4.2952461) \\
\end{array}$ & $\begin{array}{r}0.99830911 \\
(0.5838523) \\
\end{array}$ & $\begin{array}{c}0.96753201 \\
(3.6487768) \\
\end{array}$ & $\begin{array}{c}1.02917191 \\
(2.4291285) \\
\end{array}$ & $\begin{array}{l}0.98321194 \\
(2.087298) \\
\end{array}$ \\
\hline 20 & 0.5143428 & $\begin{array}{c}0.4975256 \\
(3.2696323)\end{array}$ & $\begin{array}{l}0.50439032 \\
(1.9349883)\end{array}$ & $\begin{array}{c}0.50101412 \\
(2.5914005)\end{array}$ & $\begin{array}{l}0.52050519 \\
(1.1839256)\end{array}$ & $\begin{array}{c}0.50328477 \\
(2.14993)\end{array}$ \\
\hline 50 & 0.2082766 & $\begin{array}{c}0.2028184 \\
(2.6206285)\end{array}$ & $\begin{array}{c}0.20235575 \\
(2.8427777)\end{array}$ & $\begin{array}{l}0.20426634 \\
(1.9254484)\end{array}$ & $\begin{array}{c}0.20888151 \\
(0.2895968)\end{array}$ & $\begin{array}{c}0.20375994 \\
(2.168587)\end{array}$ \\
\hline 100 & 0.1045213 & $\begin{array}{c}0.1020148 \\
(2.3980756)\end{array}$ & $\begin{array}{l}0.10122095 \\
(3.1575832)\end{array}$ & $\begin{array}{l}0.10274684 \\
(1.6976947)\end{array}$ & $\begin{array}{c}0.10448959 \\
(0.0303460)\end{array}$ & $\begin{array}{l}0.1022518 \\
(2.171328)\end{array}$ \\
\hline
\end{tabular}

TABLE 3: Comparison between MMA, FHA, SHA, FGEM, and SGEM results with exact ones and HPM responses $(\sigma=0.9)$.

\begin{tabular}{|c|c|c|c|c|c|c|}
\hline$A$ & $T_{\text {exact }}$ & $\begin{array}{c}T_{\mathrm{MMA}}=T_{\mathrm{FHA}} \\
\text { (relative error \%) }\end{array}$ & $\begin{array}{c}T_{\mathrm{FGEM}} \\
\text { (relative error \%) }\end{array}$ & $\begin{array}{c}T_{\mathrm{SHA}} \\
\text { (relative error \%) }\end{array}$ & $\begin{array}{c}T_{\mathrm{SGEM}} \\
\text { (relative error \%) }\end{array}$ & $\begin{array}{c}T_{\mathrm{HPM}}[39] \\
\text { (relative error \%) }\end{array}$ \\
\hline 0.01 & 6.2829757 & $\begin{array}{l}6.28030856 \\
(0.0424502)\end{array}$ & $\begin{array}{c}6.28297267 \\
(0.0000482)\end{array}$ & $\begin{array}{l}6.28033909 \\
(0.0419642) \\
\end{array}$ & $\begin{array}{c}6.28297267 \\
(0.0000482)\end{array}$ & $\begin{array}{c}6.2829757 \\
(0.00000005)\end{array}$ \\
\hline 0.1 & 6.2622091 & $\begin{array}{c}6.23585315 \\
(0.42087294)\end{array}$ & $\begin{array}{l}6.26202447 \\
(0.0029481)\end{array}$ & $\begin{array}{l}6.23646361 \\
(0.4111248)\end{array}$ & $\begin{array}{l}6.26203439 \\
(0.0027898)\end{array}$ & $\begin{array}{l}6.26220029 \\
(0.0001407)\end{array}$ \\
\hline 10 & 0.7727359 & $\begin{array}{c}0.75450185 \\
(2.3596739)\end{array}$ & $\begin{array}{l}0.74930385 \\
(3.032348)\end{array}$ & $\begin{array}{l}0.76075498 \\
(1.5504538)\end{array}$ & $\begin{array}{l}0.77284969 \\
(0.0147255)\end{array}$ & $\begin{array}{c}0.7564511 \\
(2.1074)\end{array}$ \\
\hline 50 & 0.1561833 & $\begin{array}{c}0.15271588 \\
(2.2200941)\end{array}$ & $\begin{array}{l}0.15087022 \\
(3.4018226)\end{array}$ & $\begin{array}{l}0.15400462 \\
(1.3949487)\end{array}$ & $\begin{array}{c}0.15573888 \\
(0.2845491)\end{array}$ & $\begin{array}{c}0.1527948 \\
(2.1696)\end{array}$ \\
\hline 100 & 0.0781394 & $\begin{array}{l}0.07642280 \\
(2.1968399)\end{array}$ & $\begin{array}{l}0.07545104 \\
(3.4404631)\end{array}$ & $\begin{array}{l}0.07706833 \\
(1.3707164)\end{array}$ & $\begin{array}{l}0.07788792 \\
(0.3218291)\end{array}$ & $\begin{array}{c}0.07644255 \\
(2.1716)\end{array}$ \\
\hline
\end{tabular}

$$
\begin{gathered}
\frac{\partial E(\dot{u}, u, t)}{\partial a} \\
=\frac{\pi}{8 \omega}\left(30 \sigma^{2} a^{5}+90 \sigma^{2} a b^{4}+180 \sigma^{2} a^{3} b^{2}\right. \\
+24 \sigma^{2} a^{2} b+16 a \omega^{4}+90 \sigma^{2} a^{2} b^{3}+48 a b^{2} \\
-32 a \omega^{2}+16 a+24 a^{3}-480 a \omega^{2} \sigma b^{2} \\
+75 \sigma^{2} a^{4} b-48 a^{3} \omega^{2} \sigma+24 a^{2} b \\
\left.-144 a^{2} \omega^{2} \sigma b+24 \sigma^{2} a^{3}+48 \sigma^{2} a b^{2}\right)=0 \\
\frac{\partial E(\dot{u}, u, t)}{\partial b \quad} \\
=\frac{\pi}{8 \omega}\left(-288 b \omega^{2}+180 \sigma^{2} a^{2} b^{3}+90 \sigma^{2} a^{4} b\right. \\
+48 \sigma^{2} a^{2} b+8 \sigma^{2} a^{3}+24 \sigma^{2} b^{3}+30 \sigma^{2} b^{5} \\
+
\end{gathered}
$$

Finally, by simultaneously solving (30) and (33) unknown parameters are determined for different values of $A$ and $\sigma$ as the second-order global error minimization method (SGEM) solution.

\section{Results}

The relative errors of the max-min, first- and second-orders of the Hamiltonian, and the global error minimization methods are shown in Tables 2, 3, and 4 for $\sigma=0.5, \sigma=0.9$, and $\sigma=1$, respectively. It is seen that the second-order global error minimization method results are closer to the exact ones than the other mentioned methods for large initial amplitudes.

The differences of obtained responses and velocities with exact ones are plotted in Figures 1 and 2, respectively. It is found that the second-order global error minimization method illustrates a better accuracy than the aforementioned techniques.

\section{Conclusion}

The Helmholtz-Duffing equation is investigated via the analytical approaches; the accuracy and validity of the obtained 
TABLE 4: Comparison between MMA, FHA, SHA, FGEM, and SGEM results with exact ones $(\sigma=1)$.

\begin{tabular}{|c|c|c|c|c|c|}
\hline$A$ & $T_{\text {exact }}$ & $\begin{array}{c}T_{\mathrm{MMA}}=T_{\mathrm{FHA}} \\
\text { (relative error \%) }\end{array}$ & $\begin{array}{c}T_{\mathrm{FGEM}} \\
\text { (relative error \%) }\end{array}$ & $\begin{array}{c}T_{\mathrm{SHA}} \\
\text { (relative error \%) }\end{array}$ & $\begin{array}{c}T_{\mathrm{SGEM}} \\
\text { (relative error \%) }\end{array}$ \\
\hline 0.01 & 6.282949702 & $\begin{array}{c}6.282949704 \\
(0.000000032)\end{array}$ & $\begin{array}{c}6.282949698 \\
(0.000000063)\end{array}$ & $\begin{array}{c}6.282953782 \\
(0.000064937)\end{array}$ & $\begin{array}{c}6.282949704 \\
(0.000000032)\end{array}$ \\
\hline 0.1 & 6.259762302 & $\begin{array}{c}6.259755078 \\
(0.000115404)\end{array}$ & $\begin{array}{c}6.259750255 \\
(0.0001924513)\end{array}$ & $\begin{array}{c}6.260158455 \\
(0.006328562)\end{array}$ & $\begin{array}{c}6.259762297 \\
(0.00000008)\end{array}$ \\
\hline 10 & 0.736288961 & $\begin{array}{l}0.720730784 \\
(2.113053139) \\
\end{array}$ & $\begin{array}{c}0.7113585054 \\
(3.385960801)\end{array}$ & $\begin{array}{l}0.7269670459 \\
(1.266067481)\end{array}$ & $\begin{array}{c}0.7337722018 \\
(0.3418167776)\end{array}$ \\
\hline 50 & 0.148282774 & $\begin{array}{l}0.1450652702 \\
(2.169843275)\end{array}$ & $\begin{array}{c}0.1431321874 \\
(3.473489510)\end{array}$ & $\begin{array}{l}0.1463367452 \\
(1.312376851)\end{array}$ & $\begin{array}{c}0.1477516413 \\
(0.3581890773)\end{array}$ \\
\hline 100 & 0.074157585 & $\begin{array}{l}0.0725471382 \\
(2.171654781)\end{array}$ & $\begin{array}{c}0.07157966046 \\
(3.476278981)\end{array}$ & $\begin{array}{l}0.0731832605 \\
(1.313856823)\end{array}$ & $\begin{array}{l}0.07389156930 \\
(0.3587167786)\end{array}$ \\
\hline
\end{tabular}

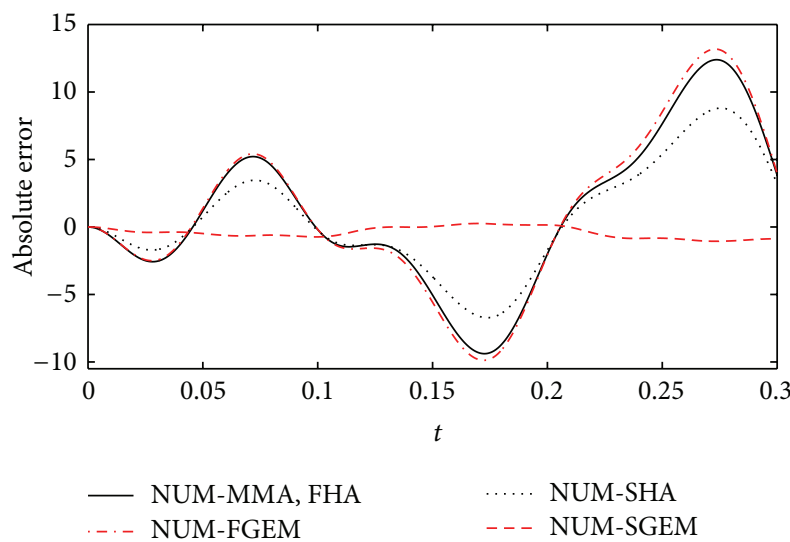

FIGURE 1: Difference between obtained responses and the numerical solution $(A=50, \sigma=0.5)$.

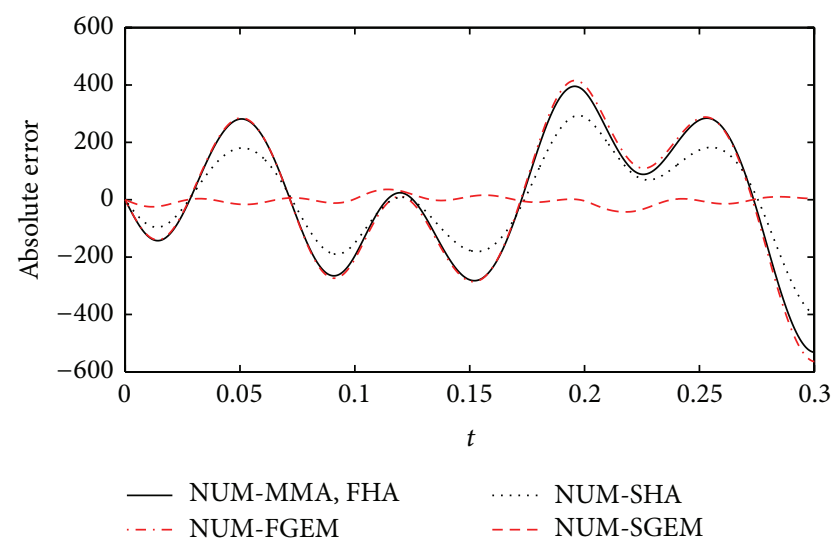

Figure 2: Difference between obtained velocities and the numerical solution $(A=50, \sigma=0.5)$.

results have been examined by comparing to the exact ones and HPM solutions. The second-order of the global error minimization method achieved better approximate solutions for this equation. In present study, it is demonstrated that higher order of the modified variational approach is accurate and simple for solving asymmetric nonlinear conservative oscillatory systems.

\section{Conflict of Interests}

The authors declare that they have no competing interests.

\section{References}

[1] A. H. Nayfeh and D. T. Mook, Nonlinear Oscillations, WileyInterscience, New York, NY, USA, 1979.

[2] S. J. Liao, Beyond Perturbation: Introduction to Homotopy Analysis Method, Chapman \& Hall/CRC Press, Boca Raton, Fla, USA, 2003.

[3] R. E. Mickens, Truly Nonlinear Oscillations: Harmonic Balance, Parametric Expansions, Iteration, and Averaging Methods, World Scientific, Singapore, 2010.

[4] I. Kovacic and M. J. Brennan, The Duffing Equation: Nonlinear Oscillators and Their Behaviour, John Wiley \& Sons, New York, NY, USA, 2011.

[5] T. Pirbodaghi, S. H. Hoseini, M. T. Ahmadian, and G. H. Farrahi, "Duffing equations with cubic and quintic nonlinearities," Computers and Mathematics with Applications, vol. 57, no. 3, pp. 500-506, 2009.

[6] Y. Khan, M. Akbarzade, and A. Kargar, "Coupling of homotopy and the variational approach for a conservative oscillator with strong odd-nonlinearity," Scientia Iranica, vol. 19, no. 3, pp. 417422, 2012.

[7] H. Askari, Z. S. Nia, A. Yildirim, M. K. Yazdi, and Y. Khan, "Application of higher order hamiltonian approach to nonlinear vibrating systems," Journal of Theoretical and Applied Mechanics, vol. 51, no. 2, pp. 287-296, 2013.

[8] S. Nourazar and A. Mirzabeigy, "Approximate solution for nonlinear Duffing oscillator with damping effect using the modified differential transform method," Scientia Iranica, vol. 20, no. 2, pp. 364-368, 2013.

[9] M. Akbarzade and A. Kargar, "Accurate analytical solutions to nonlinear oscillators by means of the Hamiltonian approach," Mathematical Methods in the Applied Sciences, vol. 34, no. 17, pp. 2089-2094, 2011.

[10] Y. Khan and A. Mirzabeigy, “Improved accuracy of He's energy balance method for analysis of conservative nonlinear oscillator," Neural Computing and Applications, vol. 25, no. 3-4, pp. 889-895, 2014.

[11] A. Mirzabeigy, M. K. Yazdi, and A. Yildirim, "Nonlinear dynamics of a particle on a rotating parabola via the analytic and semi-analytic approaches," Journal of the Association of Arab 
Universities for Basic and Applied Sciences, vol. 13, no. 1, pp. 3843, 2013.

[12] V. Marinca and N. Herisanu, "Optimal homotopy asymptotic approach to nonlinear oscillators with discontinuities," Scientific Research and Essays, vol. 8, no. 4, pp. 161-167, 2013.

[13] L. Cveticanin, M. K. Kalamiyazdi, H. Askari, and Z. Saadatnia, "Vibration of a two-mass system with non-integer order nonlinear connection," Mechanics Research Communications, vol. 43, pp. 22-28, 2012.

[14] L. Xu, "A Hamiltonian approach for a plasma physics problem," Computers and Mathematics with Applications, vol. 61, no. 8, pp. 1909-1911, 2011.

[15] Y. Khan, M. Madani, A. Yildirim, M. A. Abdou, and N. Faraz, "A new approach to van der Pol's oscillator problem," Zeitschrift fur Naturforschung Section, vol. 66, no. 10-11, pp. 620-624, 2011.

[16] Y. Khan, H. Vázquez-Leal, and N. Faraz, "An efficient new iterative method for oscillator differential equation," Scientia Iranica, vol. 19, no. 6, pp. 1473-1477, 2012.

[17] L.-L. Ke, J. Yang, and S. Kitipornchai, "An analytical study on the nonlinear vibration of functionally graded beams," Meccanica, vol. 45, no. 6, pp. 743-752, 2010.

[18] F. Alijani, F. Bakhtiari-Nejad, and M. Amabili, "Nonlinear vibrations of FGM rectangular plates in thermal environments," Nonlinear Dynamics, vol. 66, no. 3, pp. 251-270, 2011.

[19] F. Alijani, M. Amabili, K. Karagiozis, and F. Bakhtiari-Nejad, "Nonlinear vibrations of functionally graded doubly curved shallow shells," Journal of Sound and Vibration, vol. 330, no. 7, pp. 1432-1454, 2011.

[20] A. Fallah and M. M. Aghdam, "Nonlinear free vibration and post-buckling analysis of functionally graded beams on nonlinear elastic foundation," European Journal of MechanicsA/Solids, vol. 30, no. 4, pp. 571-583, 2011.

[21] P. A. Sharabiani and M. R. H. Yazdi, "Nonlinear free vibrations of functionally graded nanobeams with surface effects," Composites Part B: Engineering, vol. 45, no. 1, pp. 581-586, 2013.

[22] J. H. He, "Max-min approach to nonlinear oscillators," International Journal of Nonlinear Sciences and Numerical Simulation, vol. 9, no. 2, pp. 207-210, 2008.

[23] M. K. Yazdi, H. Ahmadian, A. Mirzabeigy, and A. Yildirim, "Dynamic analysis of vibrating systems with nonlinearities," Communications in Theoretical Physics, vol. 57, no. 2, pp. 183187, 2012.

[24] R. Azami, D. D. Ganji, H. Babazadeh, A. G. Dvavodi, and S. S. Ganji, "He's max-min method for the relativistic oscillator and high order duffing equation," International Journal of Modern Physics B, vol. 23, no. 32, pp. 5915-5927, 2009.

[25] H. M. Sedighi, K. H. Shirazi, and A. Noghrehabadi, "Application of recent powerful analytical approaches on the nonlinear vibration of cantilever beams," International Journal of Nonlinear Sciences and Numerical Simulation, vol. 13, no. 7-8, pp. 487-494, 2012.

[26] H. M. Sedighi, K. H. Shirazi, and M. A. Attarzadeh, "A study on the quintic nonlinear beam vibrations using asymptotic approximate approaches," Acta Astronautica, vol. 91, pp. 245250, 2013.

[27] J.-H. He, "Hamiltonian approach to nonlinear oscillators," Physics Letters A, vol. 374, no. 23, pp. 2312-2314, 2010.

[28] Y. Khan, Q. Wu, H. Askari, Z. Saadatnia, and M. Kalami-Yazdi, "Nonlinear vibration analysis of a rigid rod on a circular surface via hamiltonian approach," Mathematical and Computational Applications, vol. 15, no. 5, pp. 974-977, 2010.
[29] S. Durmaz, S. A. Demirbag, and M. O. Kaya, "High order Hamiltonian approach to nonlinear oscillator," International Journal of Nonlinear Sciences and Numerical Simulation, vol. 11, pp. 565-570, 2010.

[30] A. Yildirim, Z. Saadatnia, H. Askari, Y. Khan, and M. KalamiYazdi, "Higher order approximate periodic solutions for nonlinear oscillators with the Hamiltonian approach," Applied Mathematics Letters, vol. 24, no. 12, pp. 2042-2051, 2011.

[31] L. Cveticanin, M. Kalami-Yazdi, Z. Saadatnia, and H. Askari, "Application of hamiltonian approach to the generalized nonlinear oscillator with fractional power," International Journal of Nonlinear Sciences and Numerical Simulation, vol. 11, no. 12, pp. 997-1002, 2010.

[32] Y. Farzaneh and A. Akbarzadeh Tootoonchi, "Global Error Minimization method for solving strongly nonlinear oscillator differential equations," Computers \& Mathematics with Applications, vol. 59, no. 8, pp. 2887-2895, 2010.

[33] A. Mirzabeigy, M. Kalami-Yazdi, and A. Yildirim, "Analytical approximations for a conservative nonlinear singular oscillator in plasma physics," Journal of the Egyptian Mathematical Society, vol. 20, no. 3, pp. 163-166, 2012.

[34] A. Kargar and M. Akbarzade, "Analytical solution of nonlinear cubic-quintic duffing oscillator using global error minimization method," Advanced Studies in Theoretical Physics, vol. 6, no. 912, pp. 467-471, 2012.

[35] M. K. Yazdi, A. Mirzabeigy, and H. Abdollahi, "Nonlinear oscillators with non-polynomial and discontinuous elastic restoring forces," Nonlinear Science Letters A, vol. 3, pp. 48-53, 2012.

[36] Y. Khan and M. Akbarzade, "Dynamic analysis of nonlinear oscillator equation arising in double-sided driven clamped microbeam-based electromechanical resonator," Zeitschrift für Naturforschung A, vol. 67, no. 8-9, pp. 435-440, 2012.

[37] M. Akbarzade and Y. Khan, "Nonlinear dynamic analysis of conservative coupled systems of mass-spring via the analytical approaches," Arabian Journal for Science and Engineering, vol. 38, no. 1, pp. 155-162, 2013.

[38] J.-H. He, "He Chengtian's inequality and its applications," Applied Mathematics and Computation, vol. 151, no. 3, pp. 887891, 2004.

[39] A. Y. T. Leung and Z. Guo, "Homotopy perturbation for conservative Helmholtz-Duffing oscillators," Journal of Sound and Vibration, vol. 325, no. 1-2, pp. 287-296, 2009.

[40] H. Askari, Z. Saadatnia, D. Younesian, A. Yildirim, and M. Kalami-Yazdi, "Approximate periodic solutions for the Helmholtz-Duffing equation," Computers and Mathematics with Applications, vol. 62, no. 10, pp. 3894-3901, 2011.

[41] M. Akbarzade, Y. Khan, and A. Kargar, "Determination of periodic solution for the Helmholtz-Duffing oscillators by Hamiltonian approach and coupled homotopy-variational formulation," International Journal of Physical Sciences, vol. 7, pp. 560-565, 2012.

[42] Z. Li, J. Tang, and P. Cai, "A generalized harmonic function perturbation method for determining limit cycles and homoclinic orbits of Helmholtz-Duffing oscillator," Journal of Sound and Vibration, vol. 332, no. 21, pp. 5508-5522, 2013. 


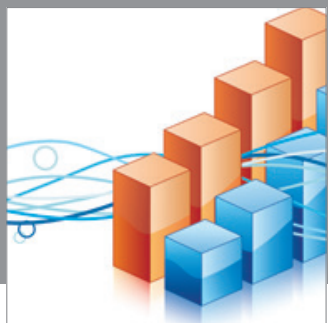

Advances in

Operations Research

mansans

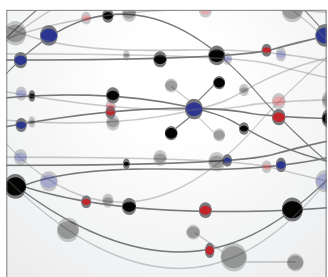

The Scientific World Journal
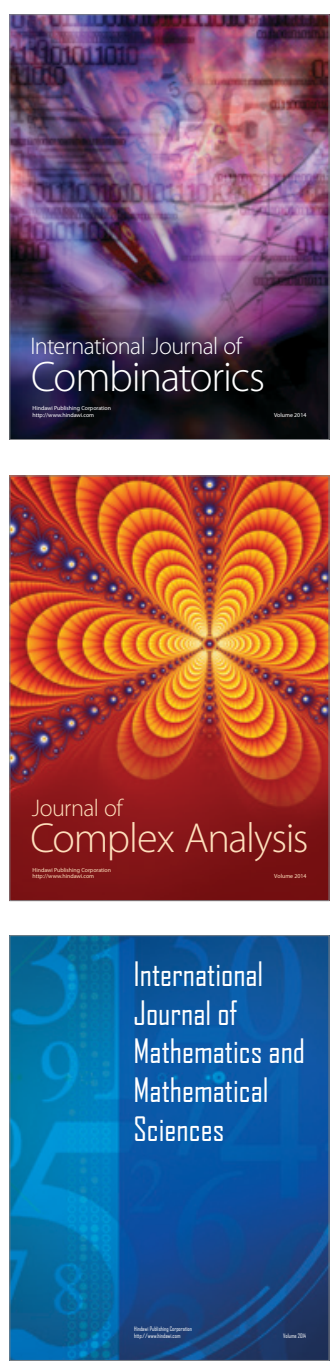
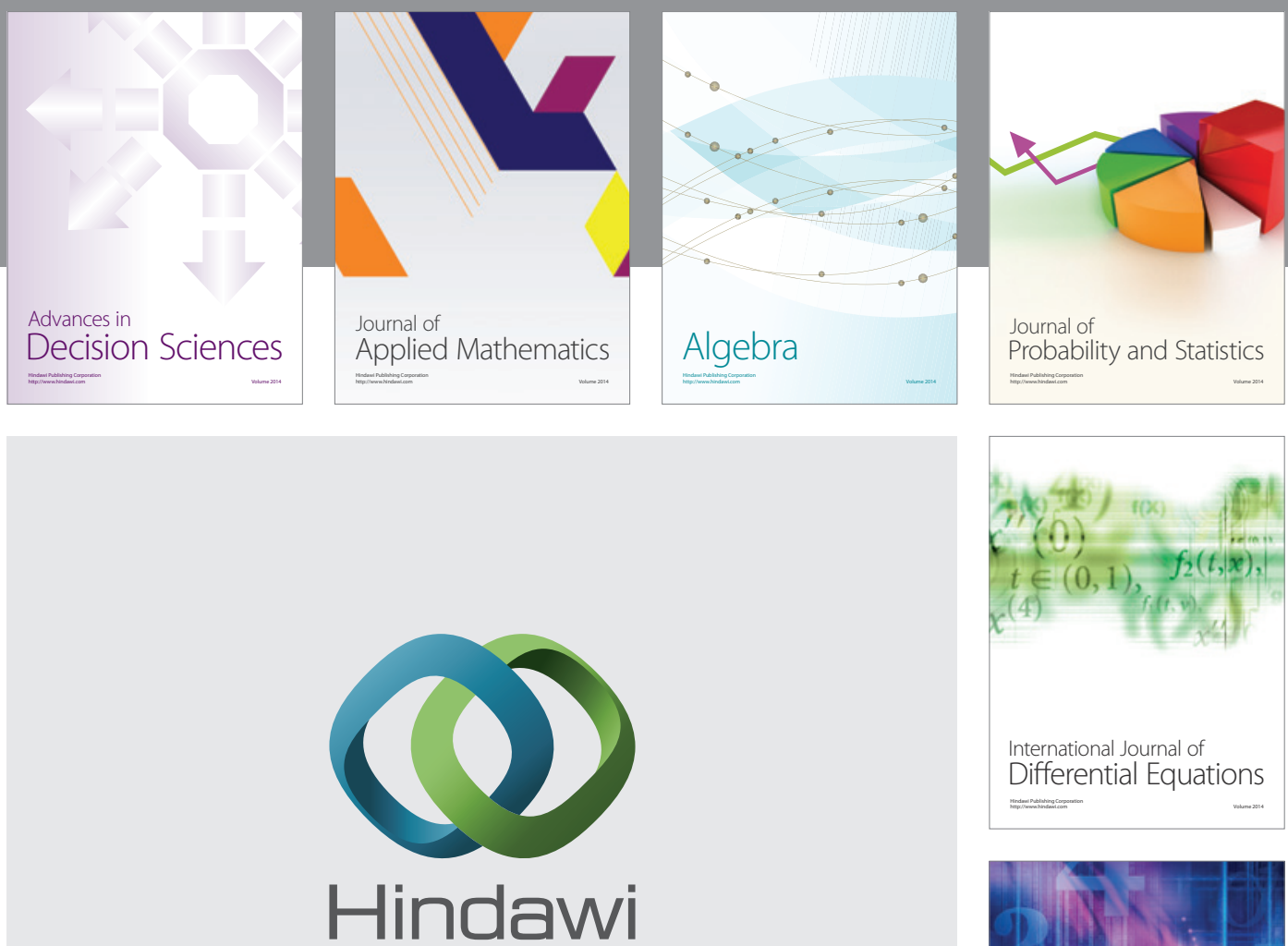

Submit your manuscripts at http://www.hindawi.com
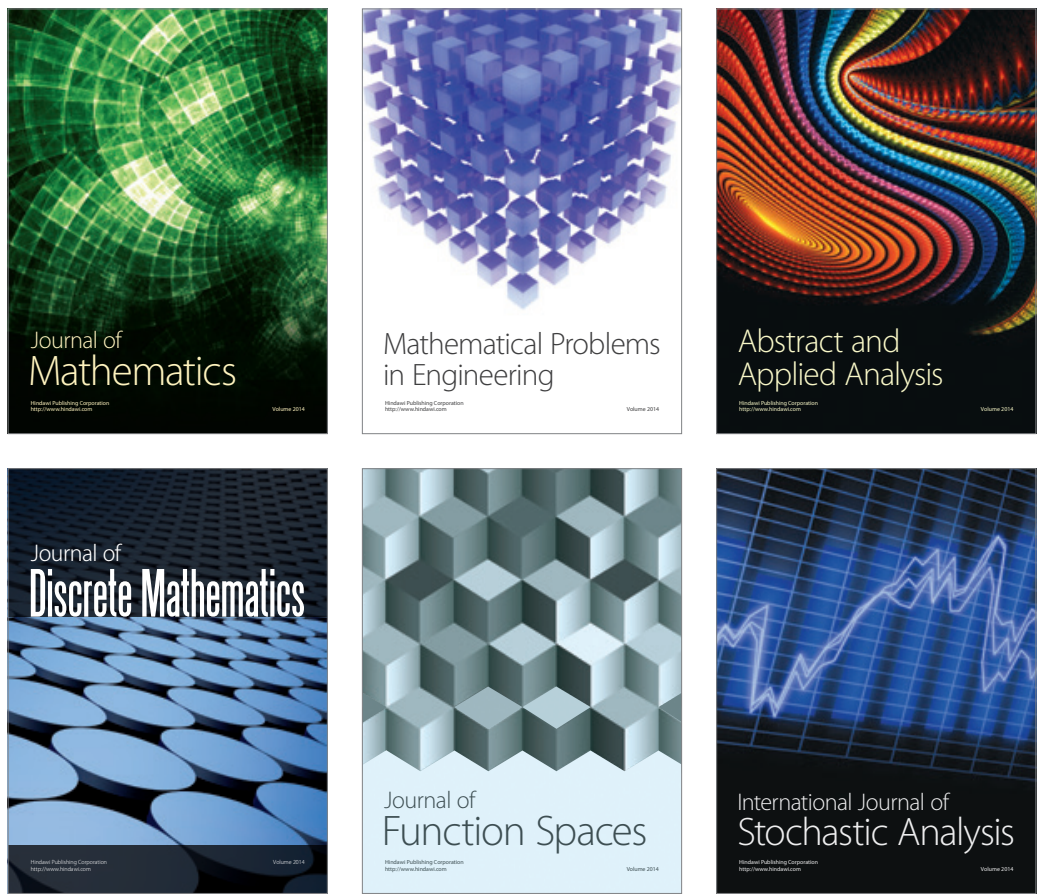

Journal of

Function Spaces

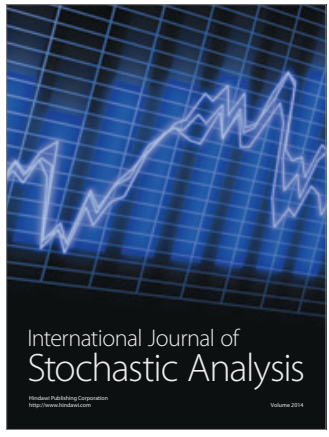

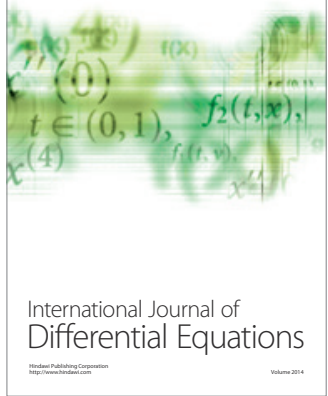
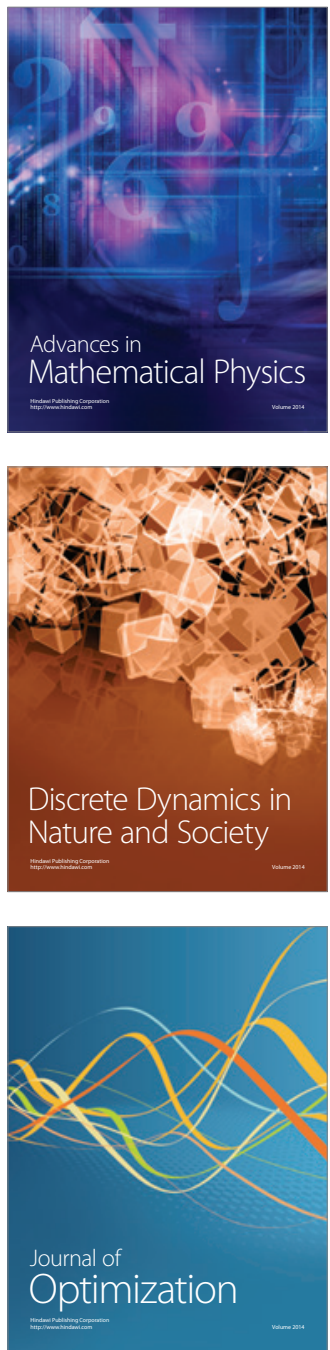